\title{
Encuesta sobre vacunación anti-rábica en veterinarios de fauna silvestre en Chile
}

\author{
Luis E. Escobar, Verónica Yung, Renzo Vargas-Rodríguez, Gonzalo Medina-Vogel y Myriam Favi
}

\section{Wildlife veterinarians rabies vaccination in Chile: a survey}

Introduction: Rabies is a lethal but preventable disease. Knowing the extent of immunization coverage among at risk populations, may help to guide immunization efforts, as well as increase the effectiveness of rabies control and prevention programs. Aim: To determine the proportion of wildlife veterinarians in Chile, as part of a group at risk of rabies transmission. Methods: An electronic survey was sent to wildlife veterinarians in Chile. Results: We found that veterinarians in Chile work mainly with carnivores and deer compared to other mammals $(p<0.001)$, rarely works with bats $(\mathrm{p}=0.6572)$. Most of the participants had been bitten by domestic animals, while a lesser proportion (29\%) by wild animals. Most of the participants never received rabies vaccination (53\%), while within the group that had started a rabies vaccination scheme, a substantial proportion (39\%) did not complete it. Discussion and conclusion: Identify the vaccination status of risk groups is important for infectious disease control and prevention programs, as this information helps to identify priority groups during outbreaks or vaccine scarcity. Wildlife veterinarians in Chile are at risk of rabies transmission and should be included in rabies prevention programs, especially considering their vulnerability and lack of biosafety practices. Increasing education in rabies epidemiology and prevention is urgently needed in veterinary faculties in Chile.

Key words: Rabies, wildlife, Chile, bat, immunization, veterinarians.

Palabras clave: Rabia, fauna de vida silvestre, Chile, murciélago, inmunización, veterinarios.

\section{Introducción}

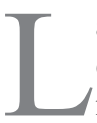
a rabia es la más mortal y una de las más antiguas enfermedades infecciosas conocidas por la humanidad $^{1}$. A la fecha, la rabia canina ha sido erradicada en varios países, pero en el mundo esta enfermedad aún es responsable de la muerte de una persona cada 15 minutos, principalmente en países de Asia y África ${ }^{1}$. Mientras, en América surgen desafíos como consecuencia del descubrimiento de nuevos reservorios de este virus ${ }^{2-5}$.

Chile ha sido declarado libre de rabia canina variantes 1 y $2^{6}$. De acuerdo a datos históricos de la Sección de Rabia del Instituto de Salud Pública de Chile (ISP), desde el año 1991, todos los virus rábicos aislados de animales han resultado ser de origen murciélago, lo que indica un ciclo silvestre de la rabia ${ }^{4}$. Cuatro variantes antigénicas (VAg) del virus rabia se han detectado en murciélagos en Chile: VAg 4 asociada a Tadarida brasiliensis, VAg 6 en el género Lasiurus, VAg de Myotis chiloensis y VAg de Histiotus macrotus ${ }^{7}$.

El virus rabia recuperado con mayor frecuencia es VAg 4 y la especie de quiróptero reservorio con mayor frecuencia de positividad en las muestras es T. brasilien$s i{ }^{7}$. Los virus aislados de casos recientes de rabia en animales domésticos (i.e., perro, gato, cerdo, vaca) fueron tipificados como VAg $4^{8}$. El actual escenario muestra una alta proporción de muestras pasivas de murciélagos positivas a virus rabia y se reporta que habitualmente murciélagos encontrados dentro de casas resultan positivos para el virus rabia9 ${ }^{9}$ Así, el riesgo de transmisión de rabia a humanos existe en Chile e incluso podría estar subestimado ${ }^{10}$.

En los Estados Unidos de América (E.U.A), donde los murciélagos insectívoros son una fuente importante de rabia al igual que en Chile ${ }^{4,5}$, los médicos veterinarios, estudiantes de veterinaria y su personal de apoyo son reconocidos como grupos con alto riesgo para contagiarse de la rabia ${ }^{11}$. De acuerdo con Advisory Committee on Immunization Practices for Human Rabies Prevention (Comité Asesor sobre Prácticas de Inmunización para la Prevención de la Rabia Humana), los grupos con alto riesgo de enfermar de rabia deben tener un esquema completo de la vacuna pre-exposición y medición de anticuerpos neutralizantes del virus de la rabia cada dos años, a fin de garantizar concentraciones plasmáticas protectoras ${ }^{11}$.

En Sudamérica, aún en el siglo XXI existe mortalidad en veterinarios por la falta de vacunación anti-rábica. Por ejemplo, en 2006 en Brasil, un veterinario de campo recogió muestras del cerebro de un herbívoro sospechoso de rabia; el veterinario no había sido vacunado contra la rabia y falleció por la infección viral ${ }^{12}$. Aun cuando desde hace 30 años los veterinarios han sido reconocidos como
State University of New York Upstate Medical University, New York, USA

Center for Global Health and Translational Science (LEE).

Universidad Andres Bello, Santiago de Chile. Facultad de Ecología y Recursos Naturales (LEE, GMV). Programa para la Conservación de Murciélagos de Chile. Departamento de Ecología y Medio Ambiente, Instituto de Filosofía y Ciencias de la Complejidad-IFICC, Santiago, Chile (LEE, RVR). Instituto de Salud Pública de Chile.

Sección Rabia (VY, MF)

Universidad de la Serena, Chile. Instituto de Ecología y

Biodiversidad, CEAZA, Departamento de Biología (RVR).

Los autores declaran que no hay conflicto de interés. Este estudio no recibió financiamiento.

Recibido: 26 de mayo de 2014 Aceptado: 7 de abril de 2015

Correspondencia a: Luis E. Escobar ecoguate2003@gmail.com 
grupo de alto riesgo para la infección con virus rabia ${ }^{13}$, en Chile poco se sabe del estado de protección inmunitaria en este grupo. En este sentido, la generación de información, dirigida a identificar grupos de riesgo y el comportamiento preventivo de éstos, es indispensable para lograr un efectivo control y prevención de enfermedades infeciosas ${ }^{14}$.

El objetivo de este estudio fue conocer el estado de vacunación anti-rábica en un grupo con riesgo en Chile a través de una encuesta. Se eligió a los veterinarios de fauna de vida silvestre debido al reconocido predominio actual del ciclo silvestre de la rabia en Chile. Considerando que Chile se encuentra libre de rabia canina, que sólo se reporta un deceso humano en las últimas dos décadas ${ }^{15}$ y que la vacunación pre-exposición no es gratuita, esperábamos que no todo el grupo de encuestados $(100 \%)$ estuviera vacunado contra la rabia.

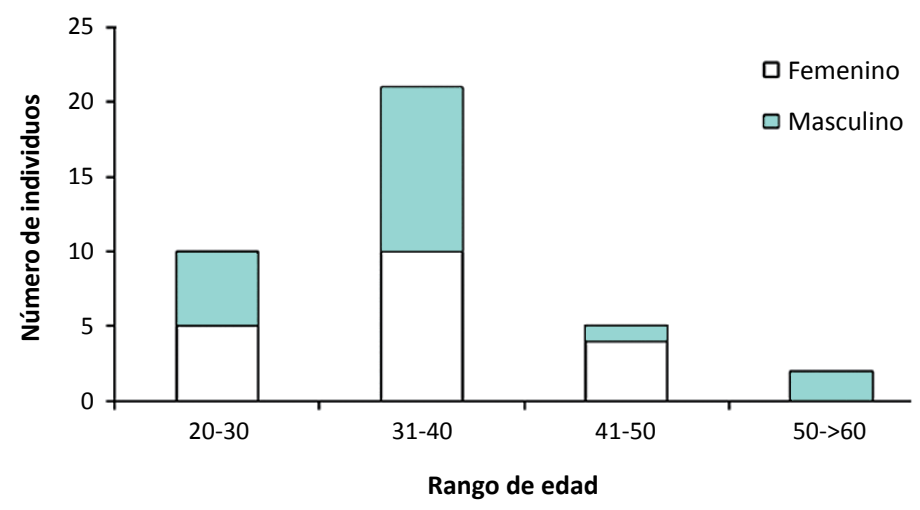

Figura 1. Distribución de veterinarios de animales silvestres encuestados según sexo y edad.

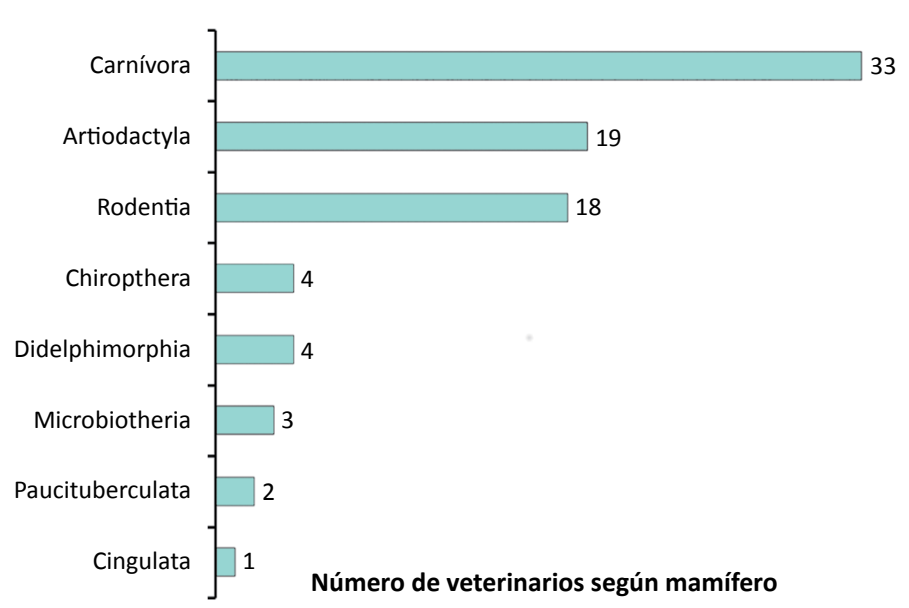

Figura 2. Órdenes taxonómicos con los que trabajan los encuestados. Eje x representa el número de veterinarios, eje y orden de mamífero con que trabaja.

\section{Métodos}

En septiembre de 2013 fue enviada una encuesta electrónica usando Google Forms (http://www.google.com/ forms/about/; (C2012 Google, Sanborn) a 107 veterinarios de fauna silvestre de Chile, miembros de la Asociación de Médicos Veterinarios Especialistas en Fauna Silvestre de Chile (AMEVEFAS). Se colectó información para una caracterización simple del grupo: género, edad, nivel de especialidad y grupo animal con que trabajaba. Se preguntó específicamente por exposición a rabia y vacunación anti-rábica pre y post-exposición. Para evaluar los grupos taxonómicos de trabajo solicitamos un detalle de las especies animales con que trabajaban y se asignaron las correspondientes familias y órdenes taxonómicos a las que pertenecían. Estos valores también fueron evaluados utilizando una prueba de $\chi^{2}$ para determinar si algunos órdenes se reportaron más que otros.

\section{Resultados}

En total, 38 veterinarios graduados ejerciendo en fauna silvestre respondieron a la encuesta $(36 \%)$. La proporción por género de la muestra fue de 1:1; no se detectó asociación de algún riesgo relacionada al género de los veterinarios. De las cuatro categorías de edad definidas, los encuestados de las categorías comprendidas entre 20 y 40 años representaron una fracción significativamente alta de la muestra $\left(82 \% ; \chi^{2}=22, \mathrm{df}=3, \mathrm{p}<0,001\right)$; no se encontraron diferencias en las categorías entre $40 \mathrm{y}>60$ años $\left(\chi^{2}=3,22, \mathrm{df}=1, \mathrm{p}=0,07\right.$ (Figura 1). La mayoría de los encuestados estudiaron la carrera en Chile (92\%) y $71 \%$ está o ha estado involucrado en un programa de postgrado (Máster o Doctorado).

Los veterinarios de fauna silvestre en Chile encuestados trabajaban con una amplia diversidad de grupos taxonómicos incluyendo peces, anfibios, reptiles, aves y mamíferos. Se observó una mayor tendencia a trabajar con mamíferos, aunque las diferencias con los otros grupos no fueron significativas $\left(\chi^{2}=6,6 ; \mathrm{gl}=4, \mathrm{p}=0,1586\right)$. En cuanto a los mamíferos, un número significativamente mayor trabajaba con los órdenes Carnívora y Artiodactyla $\left(\chi^{2}=332,7 ; \mathrm{gl}=7, \mathrm{p}<0,001\right)$. En este grupo, la mayoría $(72 \%)$ de los encuestados reportaron trabajar con al menos un orden de mamíferos, sólo cuatro encuestados reportaron trabajar con Chiroptheros (Figura 2).

Veintinueve por ciento de los encuestados reportó haber sido mordido por animales silvestres y una fracción mayor había sido mordido por perros o gatos domésticos (61\%). El 47\% de los encuestados afirma haber recibido vacunación anti-rábica; de éstos, $67 \%$ tuvo historial de vacuna pre-exposición, mientras que el resto se vacunó en respuesta a una mordedura por animal silvestre o do- 
Tabla 1. Proporción de vacunación pre- y post-exposición en veterinarios de animales silvestres con historial de mordedura. Proporción de mordidos del total de encuestados. Porcentaje de vacunación pre- y post-exposición y finalización del esquema según historial de mordedura

\begin{tabular}{|cc|}
$\begin{array}{c}\text { Antecedente de mordedura } \\
\text { según animal agresor }\end{array}$ & $\mathbf{n}$ \\
\hline Silvestre & 11 \\
Perro & 23 \\
Gato & 23 \\
\hline
\end{tabular}

\begin{tabular}{ccccc}
$\%$ & \multicolumn{2}{c}{$\begin{array}{c}\text { Vacuna pre-exposición * } \\
\%\end{array}$} & $\mathbf{n}$ & $\begin{array}{c}\text { Vacuna post-exposición* } \\
\text { * }\end{array}$ \\
29 & 3 & 27 & 3 & 27 \\
61 & 6 & 26 & 6 & 26 \\
61 & 4 & 17 & 6 & 26
\end{tabular}

No vacunados
$\mathbf{n}$
11
13

\begin{tabular}{ccc} 
\%os & $\begin{array}{c}\text { Esquema completo*\# } \\
\text { n }\end{array}$ \\
45 & 5 & 45 \\
47 & 8 & 35 \\
56 & 5 & 22 \\
\hline
\end{tabular}

\#Proporción que corresponde a los vacunados pre o post exposición.

méstico. El momento de la inmunización del profesional según el grupo de animal mordedor se resume en Tabla 1. Más de un tercio de los encuestados que recibieron vacuna no completó el esquema de vacunación (39\%). Entre los vacunados que reportaron mordedura por gato, la falla en completar las dosis requeridas del esquema alcanzó una mayor proporción (50\%). Veterinarios con historial de mordedura por mamífero silvestre tendieron a vacunarse en mayor proporción comparados con veterinarios mordidos por mamíferos domésticos (Tabla 1).

\section{Discusión}

Los resultados de este estudio de exposición al riesgo de infección laboral para rabia en médicos veterinarios de fauna de vida silvestre ha permitido la identificación de los tipos de animales con que trabajan, la frecuencia de haber sido víctimas de mordeduras por animales silvestres y domésticos para este grupo y una falla en la prevención pre y post exposición para rabia, de acuerdo a las recomendaciones actuales de inmunización.

Los veterinarios de fauna de vida silvestre en Chile parecen trabajar con una amplia diversidad de especies de vertebrados. La tendencia a trabajar más frecuentemente con mamíferos carnívoros podría exponerlos a un mayor riesgo de infección con virus de la rabia, ya que éstos, junto con los quirópteros son los principales reservorios del virus rabia ${ }^{1}$. En Chile, al menos siete especies de tres órdenes de mamíferos han sido reportados positivos a rabia, incluyendo carnívoros (zorro chilla, Lycalopex griseus; perro doméstico, Canis lupus familiaris; y gato doméstico, Felis silvestris catus), quirópteros (T. brasiliensis, M. chiloensis, Lasiurus spp. e Histiotus spp.) y Artiodactilos (bovinos, Bos taurus; y cerdos, Sus scrofa domestica $)^{4}$.

Del total de veterinarios a quienes se les envió la encuesta, únicamente $36 \%$ enviaron su respuesta. La cantidad de respuestas, aunque baja, la consideramos exitosa considerando que la tasa de respuestas para encuestas en línea varía entre 6 y $68 \%$ y que profesionales de la salud tienen una tasa de respuesta alrededor de $28 \%{ }^{18}$. El uso de encuestas en línea es una alternativa rápida, efectiva y costo-eficiente ${ }^{18}$. Nuestra encuesta fue enviada una vez por correo electrónico sin posteriores recordatorios. Reportes previos señalan que el seguimiento y recordatorios de las encuestas en línea tienen un incremento de respuestas ${ }^{19}$. Las encuestas en línea tienden a ser respondidas con mayor frecuencia por participantes jóvenes ${ }^{20}$. Aquí, la mayoría de las respuestas $(>80 \%)$ provinieron de veterinarios jóvenes del grupo etario de 20-40 años, con una pobre representación de profesionales de 41 a $>60$ años. La encuesta fue enviada a una lista actualizada de veterinarios, por lo que la falta de representación de veterinarios sobre los 40 años puede deberse a: falta de interés del grupo $>40$ años en el tópico; un limitado dominio de plataformas en línea; aspectos culturales; encuesta catalogada automáticamente como correo no deseado o pocos encuestados pertenecientes a este grupo etario. De hecho, según nuestra muestra, la población de veterinarios de fauna de vida silvestre de Chile parece estar representada por profesionales jóvenes (82\%).

Los médicos veterinarios han jugado un papel crucial en la erradicación de la rabia canina y la prevención de casos humanos de rabia transmitida por murciélagos en Chile $^{21,22}$. Sin embargo, los reportes actuales de rabia transmitida por murciélagos están aumentando ${ }^{4}$ y variantes de la rabia del murciélago han sido diagnosticadas en animales domésticos ${ }^{4,8,23}$. Tadarida brasiliensis es la especie de murciélago más abundante en Chile ${ }^{24}$. Bajo este escenario el rol de los animales domésticos en la infección a humanos es crítico en vista del alto riesgo de transmisión de rabia de murciélagos a perros, dadas las altas densidades de perros a lo largo de Chile ${ }^{25,26}$.

Los veterinarios de fauna silvestre en Chile están expuestos a las mordeduras, tanto de animales silvestres como domésticos (Tabla 1); sin embargo, menos de 50\% ha sido vacunado. Además, se hace evidente que de la fracción de individuos vacunados contra la rabia, más de un tercio no completó el esquema de vacunación. Este escenario, lleno de vacíos en el procedimiento preventivo de la enfermedad en este grupo de especialistas, hace que el riesgo de contagio de la rabia se incremente, situación que podría cambiar en Chile en el futuro cercano ya que 
desde inicios del año 2014 la ley obliga a personas dentro de los grupos de riesgo a vacunarse ${ }^{6}$. Nuestros resultados revelan el riesgo potencial de transmisión de rabia al que están expuestos los veterinarios de fauna silvestre en Chile. Así, considerando la baja tasa de inmunización de este grupo de riesgo y el incumplimiento en el esquema de vacunación, estimamos que formación en materia de bioseguridad y vacunación es urgente en las facultades y escuelas de medicina veterinaria en Chile. Los veterinarios, estudiantes de veterinaria y personal de apoyo veterinario deben ser prioridad en las campañas de vacunación y educación en la prevención de rabia, ya que son el primer frente de contacto cuando los animales enfermos se presentan al consultorio ${ }^{27}$. Las facultades de medicina veterinaria deberían incrementar los conocimientos e interés de docentes y estudiantes en esta enfermedad, incluyendo información confiable y actualizada sobre la epidemiología de rabia en Chile y el mundo. Es urgente que profesionales de la salud en Chile comprendan los riesgos de transmisión de rabia y su letalidad $(\sim 100 \%)^{1}$.

Nuestra encuesta permitió a los encuestados enviar comentarios, algunos de estos comentarios fueron interesantes. Por ejemplo, parafraseamos lo que una persona comentó: "Hace un par de años, mientras yo era estudiante de veterinaria, manejé un murciélago, que unos días más tarde resultó positivo a rabia. No me mordió. Eso fue hace mucho tiempo, así que, supongo que no voy a morir". Aún cuando los períodos de incubación de la rabia son alrededor de dos meses, la conclusión del encuestado(a) puede ser peligrosa. La literatura científica reporta largos períodos de incubación para la rabia, que van desde once meses hasta ocho años ${ }^{28}$, e incluso autores han sugerido más ${ }^{29}$. Por lo tanto, no hay un plazo límite para la vacuna post-exposición; cuando ésta se considere necesaria, debe indicarse independientemente del tiempo entre la exposición y el acceso a la consulta médica ${ }^{30}$.

Por último, la identificación de grupos de alto riesgo de infección debe ser una práctica de uso común en epidemiología como herramienta para el control y prevención de enfermedades infecciosas. La identificación y vacunación de los grupos de alto riesgo es una manera eficaz de prevenir la rabia ${ }^{11}$, además puede ser el mejor enfoque en el manejo y gestión de vacunas durante brotes o interrupción de suministros de vacuna ${ }^{27}$. El acceso a información actualizada sobre esta enfermedad es crucial para prevenir casos humanos de rabia y guiar intervenciones. Futura investigación en Chile, relacionada a inmunización de grupos de riesgo, debe incluir la estimación de la demanda de dosis de vacuna necesarias para los grupos de riesgo, los potenciales picos temporales de demanda de vacuna, áreas geográficas riesgo y la anticipación del impacto que podría generar la interrupción del suministro de vacunas contra la rabia humana. La comprensión de nuestros resultados sobre el comportamiento de profesionales en salud animal en torno al riesgo de rabia, permitió generar una aproximación de las características que deben ser tomadas en cuenta en la prevención de ésta zoonosis. Aquí basamos nuestra encuesta en veterinarios de vida silvestre por ser grupo de riesgo según los antecedentes de la enfermedad, pero otros grupos, incluyendo biólogos, podrían tener mayor contacto con murciélagos en comparación con veterinarios y debe contemplarse su situación como grupo de riesgo. Finalmente, en Chile veterinarios de fauna silvestre como grupo de riesgo carecen de buenas prácticas de bioseguridad: incluso cuando hay historial de mordedura pocos se vacunan contra rabia y los que se vacunan se vacunan mal considerando que no terminan el esquema de vacunación.

Agradecimientos: A todos los encuestados y la Asociación de Médicos Veterinarios Especialistas en Fauna Silvestre de Chile (AMVEFAS) por su colaboración en la distribución y las respuestas de esta encuesta. Agradecimientos al Laboratorio de Diagnóstico de Rabia del ISP, especialmente a Cristina Toledo, quien colaboró en la colecta de datos. RVR agradece a la Universidad de La Serena y el Instituto de Ecología y Biodiversidad. LEE agradece a UNAB por la beca para estudios de doctorado.

\section{Resumen}

Introducción: La rabia es una enfermedad infecciosa mortal pero prevenible. Establecer si existe una adecuada inmunización pre-exposición contra la rabia en un grupo de riesgo, puede ayudar a enfocar los esfuerzos de inmunización para hacer efectivos los programas de control y prevención de la enfermedad. Objetivo: Determinar mediante una encuesta la proporción de veterinarios de fauna silvestre como grupo de riesgo que han recibido inmunización contra la rabia. Métodos: $\mathrm{Se}$ realizó una encuesta electrónica a veterinarios de fauna de vida silvestre de Chile. Resultados: Los veterinarios encuestados tienden a trabajar con carnívoros y ciervos en comparación con otros mamíferos $(\mathrm{p}<0,001)$, rara vez con murciélagos $(p=0,6572)$. La mayoría de los encuestados había sido mordido por animales domésticos y una proporción menor (29\%) por animales silvestres. La mayoría nunca había sido vacunado contra la rabia (53\%), mientras que del grupo que sí había recibido vacunación anti-rábica, una importante proporción (39\%) no completó el esquema de vacunación. Sorprendentemente, este grupo de profesionales rara vez trabaja con murciélagos $(\mathrm{p}=0,6572)$, lo que puede reducir su exposición a linajes de rabia transmitida por murciélagos. Discusión y Conclusión: Identificar el estado de inmunización contra la rabia en grupos de riesgo es una información importante para evaluar los programas de prevención y control de 
enfermedades infecciosas y podría ayudar a identificar grupos prioritarios en situaciones de brote o escasez de vacuna. Los veterinarios de fauna de vida silvestre en Chile constituyen un grupo vulnerable: no mantienen prácticas de bioseguridad necesarias y tienen riesgo de adquirir la encefalitis rábica por lo que deberían ser considerados en los programas de prevención pre-exposición de la enfermedad. La educación sobre la epidemiología y prevención de la rabia es urgente en las facultades de medicina veterinaria en Chile.

\section{Referencias bibliográficas}

1.- Rupprecht C E, Hanlon C A, Hemachudha T. Rabies re-examined. Lancet Infect Dis 2002; 2 : 327-43.

2.- Vigilato M A, Cosivi O, Knöbl T, Clavijo A, Silva H M. Rabies update for Latin America and the Caribbean. Emerg Infect Dis 2013; 19: 678-9.

3.- Favoretto S R, de Mattos C C, de Mattos C A, Campos A C, Sacramento D R, Durigon E L. The emergence of wildlife species as a source of human rabies infection in Brazil. Epidemiol Infect 2013; 141: 1552-61.

4.- Favi M, Rodríguez L, Espinosa C, Yung V. Rabies in Chile: 1989-2005. Rev Chilena Infectol 2008; 25: S8-13.

5.- Blanton J D, Palmer D, Rupprecht C E. Rabies surveillance in the United States during 2009. J Am Vet Med Assoc 2010; 237: 646-57.

6.- Ministerio de Salud de Chile. Aprueba reglamento de prevención y control de rabia en el hombre y en los animales. Chile: Diario Oficial de la República de Chile; 2014: 1-7.

7.- Escobar L E, Restif O, Yung V, Favi M, Pons D J, Medina-Vogel G. Spatial and temporal trends of bat-borne rabies in Chile. Epidemiol Infect 2015; 143: 1486-94.

8.- Yung V, Favi M, Fernández J. Genetic and antigenic typing of rabies virus in Chile. Brief report. Arch Virol 2002; 147: 2197-205.

9.- Favi M, Bassaletti A, López J, Rodríguez L, Yung V. Descripción epidemiológica del reservorio de rabia en murciélagos de la Región Metropolitana, Chile 2000-2009. Rev Chilena Infectol 2011; 28: 223-8.
10.- Escobar L E, Peterson A T, Favi M, Yung V, Pons D J, Medina-Vogel G. Ecology and geography of transmission of two bat-borne rabies lineages in Chile. PLoS Negl Trop Dis 2013; 7: e2577.

11.- Manning S E, Rupprecht C E, Fishbein D, Hanlon C A, Lumlertdacha B, Guerra M, et al. Human rabies prevention-United States, 2008. MMWR Recomm Rep 2008; 57: 1-28.

12.- Brito M G, Chamone T L, da Silva F J, Wada M Y, Miranda A B, Castilho J G, et al. Antemortem diagnosis of human rabies in a veterinarian infected when handling a herbivore in Minas Gerais, Brazil. Rev Inst Med Trop Sao Paulo 2011; 53: 39-44.

13.- Centers for Disease Control. Rabies preventionUnited States, 1984. MMWR Morb Mortal Wkly Rep 1984; 33: 393-408.

14.- Frieden T R. Government's role in protecting health and safety. N Engl J Med 2013; 368 : 1857-9.

15.- Zar J. Biostatistical analysis. 5th ed. Upper Saddle River: Pearson Prentice-Hall; 1999.

16.- Fricker R D, Shonlau M. Advantages and disadvantages of Internet research surveys: Evidence from the literature. Field Methods 2002; 14: 347-67.

17.- Sheehan K B. E-mail survey response rates: a review. J Comput Mediat Commun 2006; 6. doi:10.1111/j.1083-6101.2001.tb00117.x.

18.- Kaplowitz M, Hadlock T, Levine R. A comparison of web and mail survey response rates. Public Opin Q 2004; 68: 94-101.

19.- Ernst $\mathrm{S} N$, Fabrega F. A time series analysis of the rabies control programme in Chile. Epidemiol Infect 1989; 103: 651-7.

20.- Favi M, Durán J C. Epidemiología de la rabia en
Chile (1929-1988) y perspectivas en mamíferos silvestres. Avan Cien Vet 1991; 6: 13-21.

21.- Yung V, Favi M, Fernández J. Typing of the rabies virus in Chile, 2002-2008. Epidemiol Infect 2012; 140: 2157-62.

22.- Iriarte A. Mamíferos de Chile. Santiago, Lynx Edicions; 2007.

23.- Astorga F, Poo-Muñoz D A, Escobar L E, Medina-Vogel G. In response to: "Increased dog population and potential for bat-borne rabies spillover in Chile in response to 'Dog management, abundance and potential for batborne rabies spillover in Chile' by Astorga, et al. [Prev Vet Med 118: 397-405]" by AcostaJammet G. Prev Vet Med 2015; 120: 248-9.

24.- Astorga F, Escobar L E, Poo-Muñoz D A, Medina-Vogel G. Dog ownership, abundance and potential for bat-borne rabies spillover in Chile. Prev Vet Med 2015; 118: 397-405.

25.- Lindenmayer J M, Wright J C, Nusbaum K E, Saville W J, Evanson T C, Pappaioanou M. Reported rabies pre-exposure immunization of students at US Colleges of Veterinary Medicine. J Vet Med Educ 2013; 40: 303-9.

26.- Gautret P, Carrara P, Parola P. Long incubation in imported human rabies. Ann Neurol 2014; 75: 324-5.

27.- Shankar S K, Mahadevan A, Sapico S D, Ghodkirekar M S, Pinto R G, Madhusudana S N. Rabies viral encephalitis with probable 25 year incubation period! Ann Indian Acad Neurol 2012; 15: 221-3.

28.- Ministerio de Salud. Vacuna Antirrábica de Uso Humano. Lineamientos Técnicos. Buenos Aires; 2011. 